\title{
Recent Progress on GRBs with Swift
}

\author{
N. Gehrels* and J. K. Cannizzo ${ }^{\dagger}$ \\ *Astroparticle Physics Laboratory, NASA/Goddard Space Flight Center, Greenbelt, MD 20771, \\ USA \\ ${ }^{\dagger}$ Astroparticle Physics Laboratory, CRESST/UMBC/Goddard Space Flight Center, Greenbelt, MD \\ 20771, USA
}

\begin{abstract}
We are in an exciting period of discovery for gamma-ray bursts. The Swift observatory is detecting 100 bursts per year, providing arcsecond localizations and sensitive observations of the prompt and afterglow emission. In addition, rapid-response telescopes on the ground have new capabilities to study optical emission during the prompt phase and spectral signatures of the host galaxies. The combined data set is enabling great advances in our understanding of GRBs including afterglow physics, short burst origin, and supernova physics.
\end{abstract}

Keywords: <Enter Keywords here>

PACS: $97.60 . \mathrm{Bw}, 98.70 . \mathrm{Rz}$

\section{INTRODUCTION}

GRBs are the most luminous explosions in the universe and are thought to be the birth cries of black holes. They are a product of the space age, discovered [1] by Vela and observed by satellites for 40 years. Despite impressive advances over the past three decades, the study of bursts remains highly dependent on the capabilities of the observatories which carried out the measurements. The era of the Compton Gamma Ray Observatory (CGRO) led to the discovery of more than 2600 bursts in just $9 \mathrm{yr}$. Analyses of these data produced the key result that GRBs are isotropic on the sky and occur at a frequency of roughly two per day all sky [2]. The hint from earlier instruments was confirmed that GRBs come in two distinct classes of short and long bursts, with distributions crossing at $\sim 2 \mathrm{~s}$ duration [3]. The BeppoSAX mission made the critical discovery of X-ray afterglows of long bursts [4]. With the accompanying discoveries by ground-based telescopes of optical [5] and radio [6] afterglows, long GRBs were found to emanate from star forming regions in host galaxies at typical distance of $z=1$. BeppoSAX and the following HETE-2 mission also found evidence of associations of GRBs with Type Ic SNe. This supported the growing evidence that long GRBs are caused by "collapsars" where the central core of a massive star collapses to a black hole [7].

\section{SWIFT GRBS}

Swift [8] is a dedicated GRB observatory that is now measuring many properties of the prompt and afterglow radiation. It carries a wide-field Burst Alert Telescope (BAT)

[9] that detects GRBs and positions them to arcmin accuracy, and the narrow-field X- 

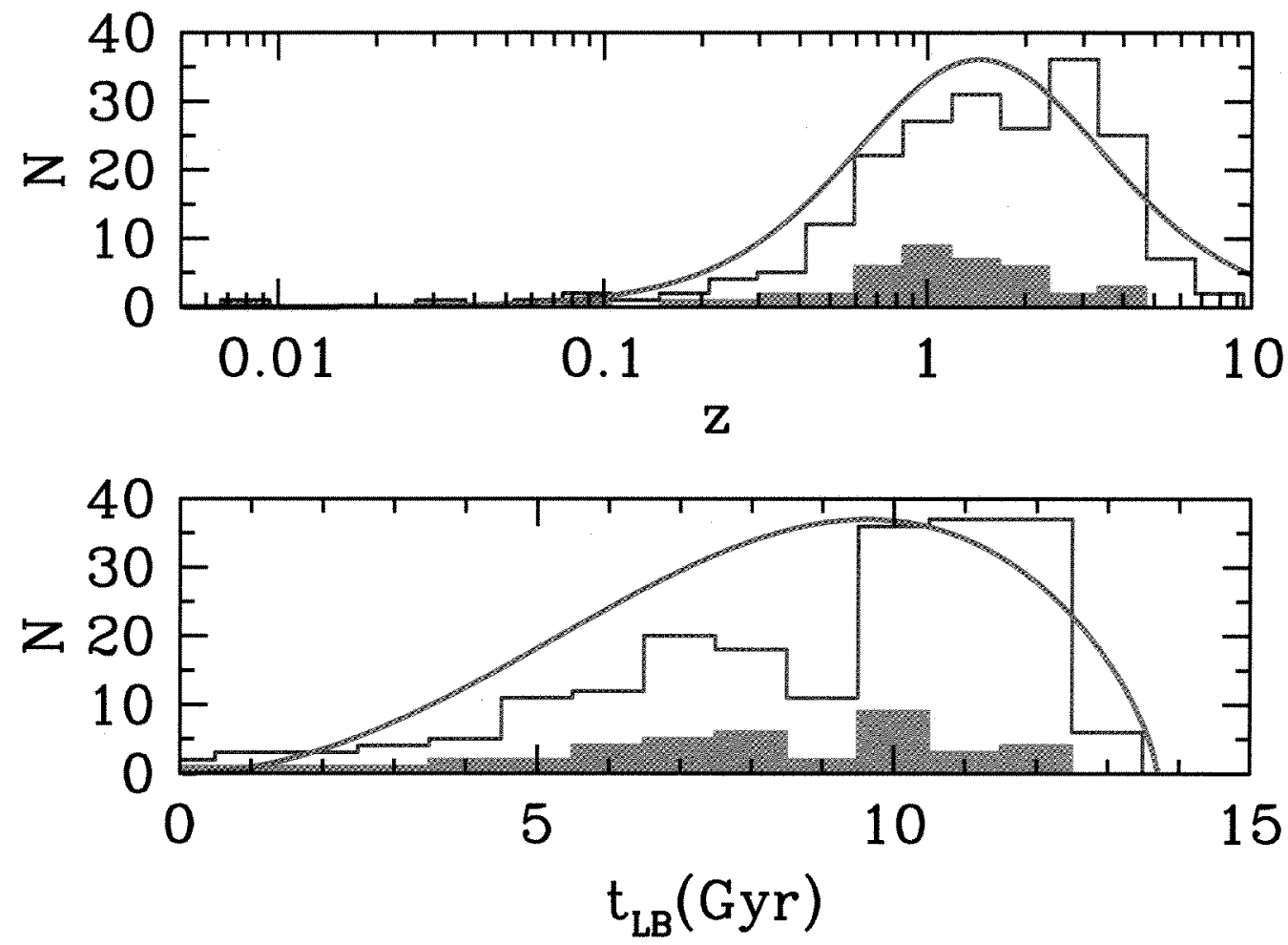

FIGURE 1. The distribution of redshifts for all GRBs to date ( $N=205$, shown in blue). The green distribution indicates the pre-Swift values $(N=41)$, the red line indicates the evolution of a comoving volume element, $(d V / d z)(1+z)^{-1}$, and the lower panel shows the distribution plotted in look-back time $t_{\mathrm{LB}}$. The factor $(1+z)^{-1}$ multiplying the comoving volume element is necessary to account for cosmological time dilation, given that the GRB rate has units volume $\mathrm{C}^{-1} \mathrm{time}^{-1}$.

Ray Telescope (XRT) [10] and UV-Optical Telescope (UVOT) [11] that observe their afterglows and determine positions to arcsec accuracy, all within $\sim 100 \mathrm{~s}$. The BAT detects the bursts in the $15-150 \mathrm{keV}$ band and determines a few-arc-min position onboard within $12 \mathrm{~s}$. The position is provided to the spacecraft which is then re-pointed to the burst location in less than 2 minutes to allow XRT and UVOT observations of the afterglow.

As of June 2010 , BAT has detected $~ 500$ GRBs. Approximately $90 \%$ of the BATdetected GRBs have repointings within 5 minutes (the remaining 10\% have spacecraft constraints that prevent rapid slewing). Of those, virtually all bursts observed promptly have detected X-ray afterglow. Already, $80 \%$ of the known X-ray afterglows are from Swift. The fraction of rapid-pointing GRBs that have UVOT detection is $\sim 30 \%$. Combined with ground-based optical observations, about $60 \%$ of Swift GRBs have optical 


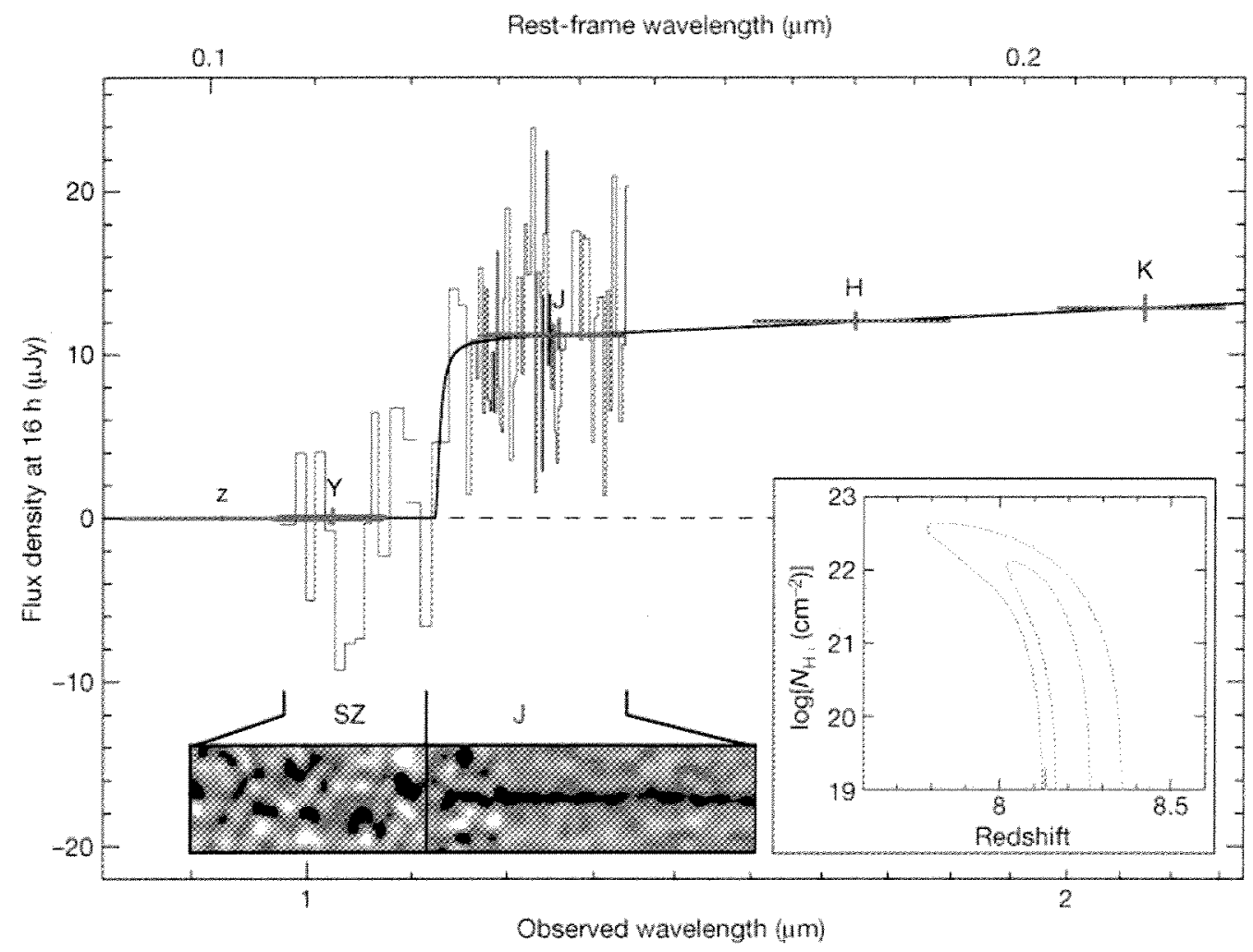

FIGURE 2. Spectra of 090432, currently the highest $z$ GRB [12]. The break, at an observed wavelength of about $1.13 \mu \mathrm{m}$, is close to the short-wavelength limit of the $J$-band spectrum, below which, although noisy, the spectrum shows no evidence of any detected continuum. A model spectrum showing the HI damping wing for a host galaxy with $N_{\mathrm{HI}}=10^{21} \mathrm{~cm}^{-2}$ at $z=8.23$ is also plotted (solid black line), and provides a good fit to the data (see also [13]).

afterglow detection. To date there are a total of $\gtrsim 200$ redshift determinations, of which 41 are pre-Swift bursts (see Figure 1).

\section{GRB PROPERTIES}

Swift is detecting GRBs at higher redshift than previous missions due to its higher sensitivity and rapid afterglow observations. The average redshift for the Swift GRBs is 2.3 compared to 1.2 for previous observations. Although statistics are poor, the highest redshift GRBs are seen to have high luminosity, resulting in fluxes well above the detection threshold. Such bursts are also strong at other wavelengths. Table 1 presents optical data for the highest redshift GRBs observed to date, where the look-back time $t_{\mathrm{LB}}(\mathrm{Gyr})$ is given in column 2. To date there are six GRBs out of $\sim 500$ at $z>5$. One might reasonably expect roughly one GRB at $z=10$ per 5 yr of Swift observations [14].

GRBs are incredibly bright. A typical galaxy at a redshift of only $z=3$ is fainter than $m \simeq 27$. Multiwavelength observations of the current record holder, GRB 090432 
TABLE 1. High $z$ GRBs.

\begin{tabular}{cccccc}
\hline$z$ & $t_{\mathrm{LB}}(\mathrm{Gyr})$ & GRB & Brightness & & \\
\hline 8.2 & 13.0 & 090423 & $K=20$ & $@$ & $20 \mathrm{~min}$ \\
6.7 & 12.8 & 080813 & $K=19$ & $@$ & $10 \mathrm{~min}$ \\
6.29 & 12.8 & 050904 & $J=18$ & $@$ & $3 \mathrm{hr}$ \\
5.6 & 12.6 & 060927 & $I=16$ & $@$ & $2 \mathrm{~min}$ \\
5.3 & 12.6 & 050814 & $K=18$ & $@$ & $23 \mathrm{hr}$ \\
5.11 & 12.5 & 060522 & $R=21$ & $@$ & $1.5 \mathrm{hr}$ \\
\hline
\end{tabular}

at $z \simeq 8$ (see Figure 2), are providing information about the universe at a time when it was only about $4 \%$ of its current age, and shed light on the process of reionization in the early universe $[12,13]$. A study of the damped Lyman $-\alpha$ systems (DLAs) associated with optical spectra of GRBs and their hosts has provided detailed information on the metallicity history of the universe, and allowed a comparison of the metallicity history inferred from similar studies involving QSOs (see Figure 3).

GRBs come in two kinds, long and short, where the dividing line between the two is $\sim 2 \mathrm{~s}$ [3]. A further division can be made spectrally according to their hardness ratio (i.e., ratio of high to low energies). The redshift range is from about 0.2 to 2 for short GRBs (sGRBs), with a mean of about 0.4. For long GRBs (lGRBs) the range is between about 0.009 and 8.2 , with a mean of about 2.3. The typical energy release is $\sim 10^{49}-10^{50}$ erg for sGRBs and $\sim 10^{50}-10^{51}$ erg for lGRBs. These ranges are based on observed isotropic-equivalent energies of $\sim 10^{51}$ erg for sGRBs and $\sim 10^{53}$ erg for lGRBs, and estimates for jet beaming for each class, $\theta_{\mathrm{j}} \sim 5^{\circ}$ for lGRBs and $\theta_{\mathrm{j}} \sim 15^{\circ}$ for sGRBs $[17,18]$. Beaming angles for sGRBs are still highly uncertain. The corresponding beaming factors $f_{b}=1-\cos \theta_{\mathrm{j}} \simeq \theta_{\mathrm{j}}^{2} / 2$ are roughly $1 / 300$ for 1 GRBs and $1 / 30$ for sGRBs. Figure 4 shows a comparison of the X-ray afterglow fluxes at $11 \mathrm{hr}$ post-GRB with the isotropic-equivalent gamma-ray energies. The $L_{\mathrm{X}} / E_{\gamma \text {-iso }}$ values are similar between IGRBs and sGRBs. The sGRBs have weaker X-ray afterglows, a mean value of $\sim 7 \times 10^{-10} \mathrm{erg} \mathrm{cm}^{-2} \mathrm{~s}^{-1}$ versus $\sim 3 \times 10^{-9} \mathrm{erg} \mathrm{cm}^{-2} \mathrm{~s}^{-1}$ for lGRBs.

\section{SHORT GRBS}

At Swift's launch, the greatest mystery of GRB astronomy was the nature of shortduration, hard-spectrum bursts. Although more than 50 lGRBs had afterglow detections, no afterglow had been found for any sGRB. In May 2005 (GRB 050509B), Swift provided the first sGRB X-ray afterglow localization [20]. This burst plus the HETE2 GRB 050709 and Swift GRB 050724 led to a breakthrough in our understanding $[20,21,22,23,24,25,26]$ of short bursts. BAT has now detected 52 short GRBs, of which 41 have XRT detections and 17 optical detections. There are 11 firm redshifts (see Table 2). An additional three sGRBs have been detected by HETE-2, one by INTEGRAL, and one by $\mathrm{Fermi} / \mathrm{LAT}$.

sGRBs encompass a smaller redshift range, have weaker afterglows, smaller $E_{\mathrm{iso}}$, and are found in all galaxy types. In stark contrast to IGRBs, sGRBs can originate from 


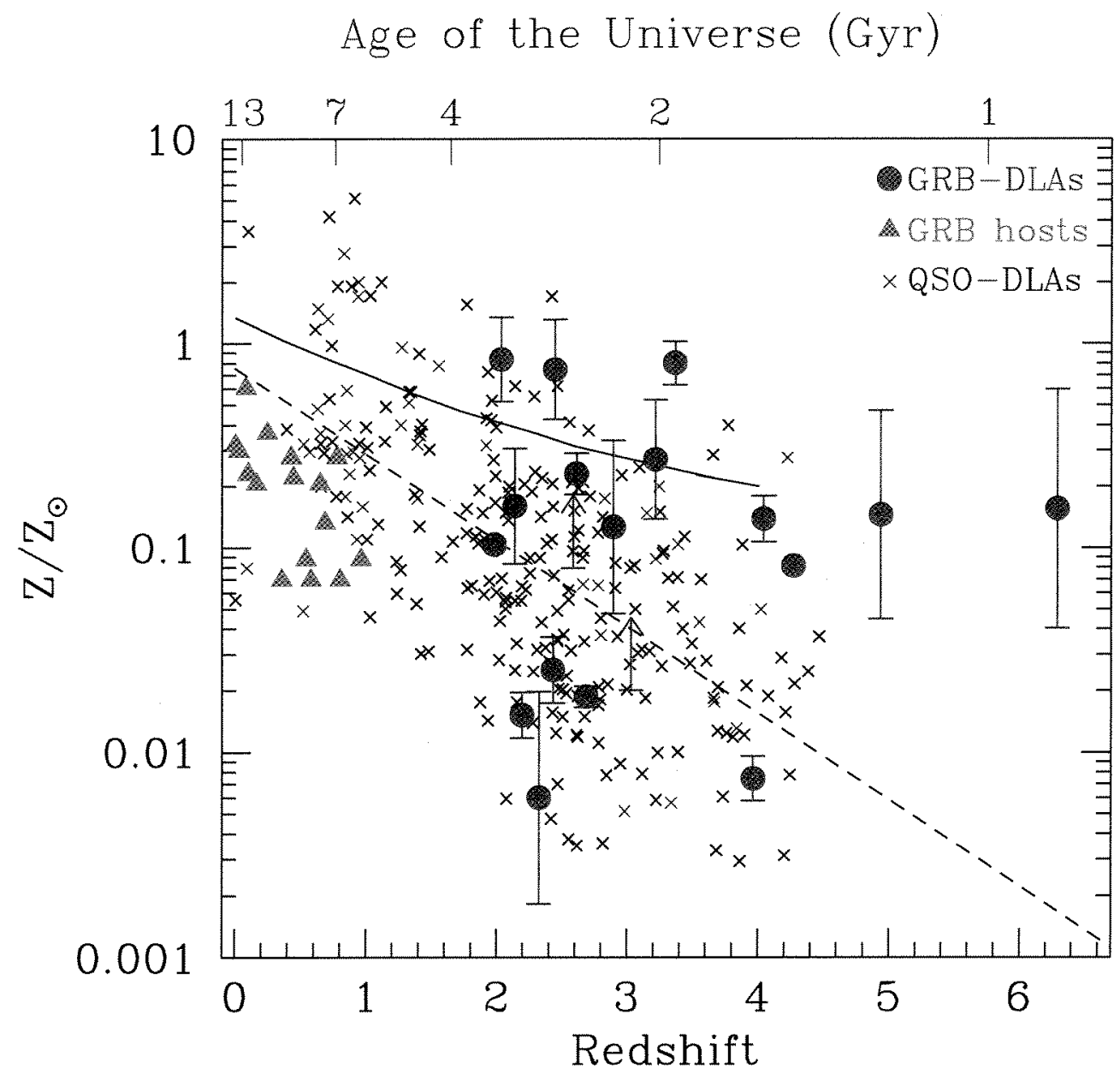

FIGURE 3. Redshift evolution of the metallicity relative to solar values determined from DLAs for 17 GRB-DLAs at $z>2,16$ GRB hosts at $z<1$ and $\sim 250$ QSO-DLAs in the interval $0<z<4.4$ [15]. The dashed line is the best fit linear correlation for QSO-DLAs. The solid line is the mean metallicity predicted by semi-analytic models for galaxy formation [16]. The GRB-DLAs metallicity for $2<z<4.5$ is $\sim 2.5 \times$ higher than the average value for QSO-DLAs in the same interval.

regions with low star formation rate. GRB 050509B and 050724 were from elliptical galaxies with low current star formation rates while GRB 050709 was from a region of a star forming galaxy with no nebulosity or evidence of recent star formation activity in that location. Recent HST observations of locations of sGRBs in their hosts reveal that short bursts trace the light distribution of their hosts while long bursts are concentrated in the brightest regions [27]. sGRBs are also different from 1GRBs in that accompanying supernovae are not detected for nearby events $[21,22,24]$. Taken together, these results support the interpretation that short bursts are associated with an old stellar population, and may arise from mergers of compact binaries [i.e., double neutron star or neutron star 


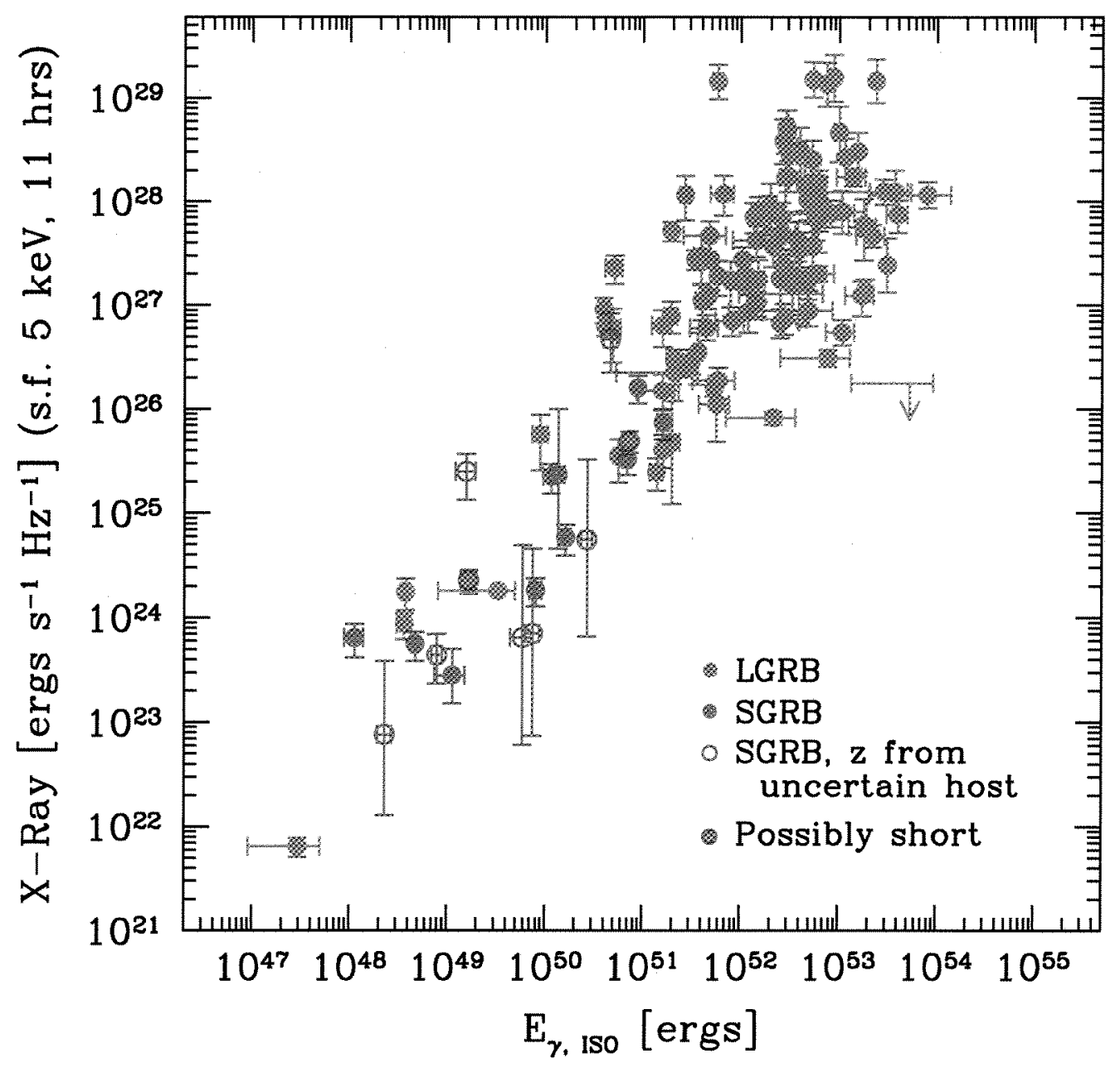

FIGURE 4. A comparison of the afterglows of sGRBs and IGRBs [19]. The $y$-axis shows the $5 \mathrm{keV}$ flux values at $T 0+11 \mathrm{hr}$, where $T 0$ indicates the BAT GRB trigger time, while the $x$-axis indicates the isotropic-equivalent $\gamma$-ray energies determined from the prompt emission.

- black hole (NS-BH) binaries].

\section{GRB-SN CONNECTION}

On 18 February 2006 Swift detected the remarkable burst GRB 060218 that provided considerable new information on the connection between SNe and GRBs. It lasted longer than and was softer than any previous burst, and was associated with SN 2006aj at only $z=0.033$. The BAT trigger enabled XRT and UVOT observations during the prompt phase of the GRB and initiated multiwavelength observations of the supernova from the time of the initial core collapse. 
TABLE 2. Short GRBs with redshifts.

\begin{tabular}{llcccccc}
\hline Name & $z$ & Host & $\begin{array}{c}\text { Fluence }(\gamma-\text { ray }) \\
\left(10^{-8} \mathrm{erg} \mathrm{cm}^{-2}\right)\end{array}$ & $\begin{array}{c}\text { Duration } \\
(\mathrm{s})\end{array}$ & $\begin{array}{c}\text { Spectral } \\
\text { Lag }(\mathrm{ms})\end{array}$ & EE* $^{*}$ & Comment \\
\hline $050509 \mathrm{~B}$ & 0.225 & elliptical & $0.95 \pm 0.25$ & 0.03 & $4.7 \pm 3.2$ & $\mathrm{~N}$ & low SF, HST \\
050724 & 0.258 & elliptical & $39 \pm 10$ & 3.0 & $-4.2 \pm 8.2$ & $\mathrm{Y}$ & low SF, HST \\
$051221 \mathrm{~A}$ & 0.5465 & SF galaxy & $116 \pm 4$ & 1.4 & $0 \pm 0.4$ & $\mathrm{~N}$ & $\mathrm{HST}$ \\
061006 & 0.4377 & galaxy & $143 \pm 14$ & $0.5(130)$ & $9.2 \pm 7.1$ & $\mathrm{Y}$ & $\mathrm{HST}$ \\
$070429 \mathrm{~B}$ & 0.904 & galaxy & $6.3 \pm 1$ & 0.5 & $7 \pm 5$ & $\mathrm{~N}$ & $4 \mathrm{kpc}, \mathrm{spec} z$ \\
$070714 \mathrm{~B}$ & 0.923 & galaxy & $72 \pm 9$ & $2.9(64)$ & $14 \pm 7$ & $\mathrm{Y}$ & $R \simeq 25.5, \operatorname{spec} z$ \\
070724 & 0.457 & galaxy & $3 \pm 0.7$ & 0.4 & $22 \pm 35$ & $\mathrm{~N}$ & SF host @ 2" \\
071227 & 0.383 & galaxy & $22 \pm 3$ & 0.18 & $4 \pm 14$ & $\mathrm{Y}$ & \\
090426 & 2.6 & & 18 & 1.2 & $1.7 \pm 12.5$ & $\mathrm{~N}$ & long or short? \\
090510 & 0.903 & & $34 \pm 4$ & 0.3 & $0.8 \pm 1.0$ & $\mathrm{~N}$ & LAT \\
090927 & 1.37 & & $22 \pm 3$ & 2.2 & & $\mathrm{~N}$ & \\
\hline
\end{tabular}

* EE: extended emission

The spectral peak in prompt emission at $\sim 5 \mathrm{keV}$ places GRB 060218 in the X-ray flash category of GRBs [28], the first such association for a GRB-SN event. Combined BAT-XRT-UVOT observations provided the first direct observation of shock-breakout in a SN [28]. This is inferred from the evolution of a soft thermal component in the Xray and UV spectra, and early-time luminosity variations. Concerning the supernova, $\mathrm{SN}$ 2006aj was dimmer by a factor $\sim 2$ than the previous SNe associated with GRBs, but still $\sim 2-3$ times brighter than normal SN Ic not associated with GRBs [29, 30]. Recently the super-long under-luminous burst $100316 \mathrm{D}$ at $z=0.059$ was found to be associated with SN 2010bh [31, 32]. Both the spectral and temporal behavior of this burst are similar to GRB 060218, except that the associated SN 2006aj was less energetic than SN 2010bh. GRB 100316D is an important addition to the currently sparse sample of spectroscopically confirmed GRB-SNe.

GRB 060218 was an underluminous burst, as were two of the other three previous cases. Because of the low luminosity, these events are only detected when nearby and are therefore rare occurrences. However, they are actually $\sim 5-10$ times more common in the universe than normal GRBs [33].

\section{CONCLUSIONS}

The future is bright for GRB astronomy. Swift should maintain its orbit for at least ten years, and possibly much longer. Swift short GRBs are found to reside in demonstrably different environments than long GRBs and are not accompanied by SNe. For instance, their spatial distributions over their host galaxies are much more spread out [27]. The weight of the evidence lends credence to the NS-NS merger model. Distant GRBs are elucidating the properties of the high $-z$ universe and probing into the era of reionization. In spite of the rich progress in the field, several open questions remain: What are the detailed properties of short GRBs? Do GRBs accompany the demise of the earliest stars? What are physical processes that take place at the center of collapsing massive stars to occasionally produce IGRBs? 


\section{REFERENCES}

1. R. W. Klebesadel, I. B. Strong, and R. A. Olson, ApJL 182, L85-L88 (1973).

2. C. A. Meegan, G. J. Fishman, R. B. Wilson, J. M. Horack, M. N. Brock, et al., Nature 355, 143-145 (1992).

3. C. Kouveliotou, C. A. Meegan, G. J. Fishman, N. P. Bhat, M. S. Briggs, et al., ApJL 413, L101-L104 (1993).

4. E. Costa, F. Frontera, J. Heise, M. Feroci, J. in't Zand, et al., Nature 387, 783-785 (1997).

5. J. van Paradijs, P. J. Groot, T. Galama, C. Kouveliotou, R. G. Strom, et al., Nature 386, 686-689 (1997).

6. D. A. Frail, S. R. Kulkarni, L. Nicastro, M. Feroci, and G. B. Taylor, Nature 389, 261-263 (1997).

7. A. I. MacFadyen, and S. E. Woosley, ApJ 524, 262-289 (1999).

8. N. Gehrels, G. Chincarini, P. Giommi, K. O. Mason, J. A. Nousek, et al., ApJ 611, 1005-1020 (2004).

9. S. D. Barthelmy, L. M. Barbier, J. R. Cummings, E. E. Fenimore, N. Gehrels, et al., Space Science Reviews 120, 143-164 (2005).

10. D. N. Burrows, J. E. Hill, J. A. Nousek, J. A. Kennea, A. Wells, et al., Space Science Reviews 120, 165-195 (2005).

11. P. W. A. Roming, T. E. Kennedy, K. O. Mason, J. A. Nousek, L. Ahr, et al., Space Science Reviews 120, 95-142 (2005).

12. N. R. Tanvir, D. B. Fox, A. J. Levan, E. Berger, K. Wiersema, et al, Nature 461, 1254-1257 (2009).

13. R. Salvaterra, M. Della Valle, S. Campana, G. Chincarini, S. Covino, et al., Nature 461, 1258-1260 (2009).

14. N. R. Butler, J. S. Bloom, and D. Poznanski, ApJ 711, 495-516 (2010).

15. S. Savaglio, "The Cosmic Chemical Evolution as seen by the Brightest Events in the Universe," in IAU Symposium, edited by K. Cunha, M. Spite, \& B. Barbuy, 2010, vol. 265 of IAU Symposium, pp. 139-146.

16. R. S. Somerville, J. R. Primack, and S. M. Faber, MNRAS 320, 504-528 (2001).

17. D. N. Burrows, D. Grupe, M. Capalbi, A. Panaitescu, S. K. Patel, et al., ApJ 653, 468-473 (2006).

18. D. Grupe, D. N. Burrows, S. K. Patel, C. Kouveliotou, B. Zhang, et al., ApJ 653, $462-467$ (2006).

19. M. Nysewander, A. S. Fruchter, and A. Pe'er, ApJ 701, 824-836 (2009).

20. N. Gehrels, C. L. Sarazin, P. T. O'Brien, B. Zhang, L. Barbier, et al., Nature 437, 851-854 (2005).

21. J. S. Bloom, J. X. Prochaska, D. Pooley, C. H. Blake, R. J. Foley, et al., ApJ 638, 354-368 (2006).

22. D. B. Fox, D. A. Frail, P. A. Price, S. R. Kulkarni, E. Berger, et al., Nature 437, 845-850 (2005).

23. J. S. Villasenor, D. Q. Lamb, G. R. Ricker, J. Atteia, N. Kawai, et al., Nature 437, 855-858 (2005).

24. J. Hjorth, D. Watson, J. P. U. Fynbo, P. A. Price, B. L. Jensen, et al., Nature 437, 859-861 (2005).

25. S. D. Barthelmy, G. Chincarini, D. N. Burrows, N. Gehrels, S. Covino, et al., Nature 438, 994-996 (2005).

26. E. Berger, P. A. Price, S. B. Cenko, A. Gal-Yam, A. M. Soderberg, et al., Nature 438, 988-990 (2005).

27. W. Fong, E. Berger, and D. B. Fox, ApJ 708, 9-25 (2010).

28. S. Campana, V. Mangano, A. J. Blustin, P. Brown, D. N. Burrows, et al., Nature 442, 1008-1010 (2006).

29. E. Pian, P. A. Mazzali, N. Masetti, P. Ferrero, S. Klose, et al., Nature 442, 1011-1013 (2006).

30. P. A. Mazzali, J. Deng, K. Nomoto, D. N. Sauer, E. Pian, et al., Nature 442, 1018-1020 (2006).

31. R. L. C. Starling, K. Wiersema, A. J. Levan, T. Sakamoto, D. Bersier, et al., ArXiv e-prints (2010), 1004.2919.

32. Y. Fan, B. Zhang, D. Xu, E. Liang, and B. Zhang, ArXiv e-prints (2010), 1004.5267.

33. A. M. Soderberg, S. R. Kulkarni, E. Nakar, E. Berger, P. B. Cameron, et al., Nature 442, 1014-1017 (2006). 